\title{
ESTUDO COMPARATIVO ENTRE A APLICAÇÃO DE CRIOTERAPIA, CINESIOTERAPIA E ONDAS CỦRTAS NO TRATAMENTO DA OSTEOARTRITE DE JOELHO
}

\author{
COMPARISON OF CRYOTHERAPY, EXERCISE AND SHORT WAVES FOR IN KNEE \\ OSTEOARTHRITIS TREATMENT
}

\author{
Adriana lucia Pastore Silva ${ }^{1}$, Daniela Mayumi Imoto ${ }^{2}$, Alberto Tesconi Croc ${ }^{3}$
}

\begin{abstract}
RESUMO
A osteoartrite é a forma mais prevalente de doença articular. Os agentes físicos como o gelo e o calor podem combater o processo álgico quando corretamente indicados e utilizados. O objetivo deste trabalho foi a comparação de protocolos de tratamento fisioterapêutico, que envolveram o uso de cinesioterapia, crioterapia e ondas curtas, em indivíduos com osteoartrite de joelho. Em um estudo randomizado prospectivo cego foram tratados 25 indivíduos (25 joelhos), de 58 a 78 anos. GRUPO A: cinesioterapia e ondas curtas $(n=9)$. GRUPO B: cinesioterapia e gelo $(n=6)$. GRUPO C: cinesioterapia (grupo controle) $(n=9)$. As variáveis analisadas foram: percepção subjetiva de dor (Borg), qualidade funcional (Lequesne), amplitude de movimento, flexibilidade e força muscular. A dor melhorou significativamente somente no grupo B, com qualidade funcional e flexibilidade positiva em todos grupos. $\mathrm{O}$ ganho de amplitude foi semelhante nos grupos B e C. Houve manutenção da força flexora nos grupos $\mathrm{A}$ e $\mathrm{B}$ e ganho nos indivíduos do grupo C. Na musculatura extensora observou-se ganho de força no grupo B e $\mathrm{C}$ e perda no grupo A. O melhor protocolo foi aquele que envolveu a aplicação de gelo e cinesioterapia para analgesia; não houve relação de ganho de amplitude, flexibilidade e força associado a termoterapia.
\end{abstract}

Descritores: Osteoartrite de joelho; Crioterapia; Ondas curtas; Fisioterapia

Citação:Silva ALP, Imoto DM, Croci AT. Estudo comparativo entre a aplicação de crioterapia, cinesioterapia e ondas curtas no tratamento da osteoartrite de joelho. Acta Ortop Bras. [periódico na Internet]. 2007; 15(4):204-209. Disponível em URL: http://www.scielo.br/aob.

\section{INTRODUÇÃO}

A osteoartrite $(\mathrm{OA})$ é uma das doenças mais comuns do sistema esquelético e pode ser definida como uma condição degenerativa que afeta as articulações sinoviais ${ }^{(1)}$, sendo a forma mais prevalente de doença articular que não leva a acometimento sistêmico, sem mortalidade associada(2).

A OA do joelho é uma doença de caráter inflamatório e degenerativo que provoca a destruição da cartilagem articular e leva a uma deformidade da articulação(3).

A dor, geralmente, é o primeiro fator incapacitante da OA, levando posteriormente a alterações articulares, periarticulares e disfunção

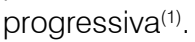

\section{SUMMARY}

Osteoarthritis is the most prevalent form of joint disease. Physical agents such as ice and heat can fight the pain process, when correctly indicated and used. The objective of this study was to compare physiotherapy protocols involving the use of exercise, cryotherapy and short waves in individuals with knee osteoarthritis. In a prospective randomized study, 25 individuals were treated (25 knees), with ages ranging from 58 to 78 years. GROUP A: exercise and short-waves $(n=9)$. GROUP B: exercise and ice $(n=6)$. GROUP C: exercise alone (control) $(n=9)$. Analyzed variables were: subjective perception of pain (Borg), functional quality (Lequesne), range of motion, flexibility and muscular strength. Pain significantly improved only for group $\mathrm{B}$, with positive functional quality and flexibility in all groups. The improvement in range of motion was similar for groups $\mathrm{B}$ and $\mathrm{C}$. Flexion strength was maintained for groups $\mathrm{A}$ and $\mathrm{B}$, and strength gain for individuals in group $\mathrm{C}$. In the extensors muscles, strength gain was seen in groups $B$ and $C$, while strength loss was reported for group $A$. The best protocol was that one involving cryotherapy and exercises for pain relief. All groups showed improved functional quality; no correlation was found for range of motion gain, flexibility and strength gain associated with thermotherapy.

Keywords: Osteoarthritis of the knee; Cryotherapy; Short wave; Physiotherapy

Citation: Silva ALP, Imoto DM, Croci AT. Comparison of cryotherapy, exercise and short waves for in knee osteoarthritis treatment. Acta Ortop Bras. [serial on the Internet]. 2007; 15(4): 204-209. Available from URL: http://www.scielo.br/aob.

Os agentes físicos podem combater o processo álgico, quando corretamente indicados e utilizados. Dentre estes agentes destacam-se a crioterapia, o calor superficial e profundo(1)

Embora a OA esteja relacionada com fatores mecânicos de sobrecarga articular levando à lesão cartilaginosa a atividade física regular e dosada pode melhorar algumas das alterações secundárias relacionadas com esta doença(1)

O tratamento da OA deve ser continuado, baseando-se em terapia medicamentosa e física ${ }^{(2)}$

A literatura de monstra interesse nas comprovações científicas das propostas de medicina física, até o momento muitas vezes baseadas na observação e empirismo. As revisões tentam demonstrar o efeito be-

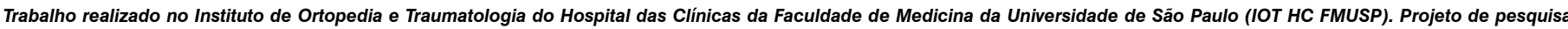
aprovado pela Comissão de ética da FMUSP

Endereço para correspondência: Rua Tamandaré, 734 ap 154 bloco A - Liberdade - São Paulo - SP - Cep: 01525-000 - E-mail: adriana pastore@hotmail.com

1. Fisioterapeuta, Mestre em Ciências na área de Ortopedia e Traumatologia pelo Instituto de Ortopedia e Traumatologia do HC/FMUSP.

2. Fisioterapeuta do Instituto de Ortopedia e Traumatologia do HC/FMUSP

3. Professor Associado do Departamento de Ortopedia e Traumatologia e Coordenador do Banco de Tecidos do IOT/ HC/FMUSP 
néfico de certas técnicas, porém várias comprovações são necessárias sobre a real utilidade de cada método ou aparelho seja proposto(2). O objetivo deste trabalho foi a comparação de protocolos de tratamento fisioterapêutico, que envolvem o uso de cinesioterapia, crioterapia e uma forma de calor profundo (ondas curtas), em pacientes com diagnóstico de OA de joelho atendidos no ambulatório do Instituto de Ortopedia e Traumatologia do Hospital das Clinicas da Faculdade de Medicina da Universidade de São Paulo.

\section{CASUÍSTICA}

Foram analisados 25 pacientes sedentários (19 do sexo feminino e 6 do sexo masculino) num total de 25 joelhos (15 direitos e 10 esquerdos) no período de Maio a Dezembro de 2003, com diagnóstico de osteoartrite primária do joelho, cuja idade variou de 58 a 78 anos (média de 67,56 anos), peso corporal entre 53 e $136 \mathrm{~kg}$ (média de 76,16kg), altura entre 1,50 e 1,85 m (média 1,60m), encaminhados do Grupo de Artroplastias da Disciplina de Ortopedia Geral do IOT HC FMUSP.

\section{Os critérios de inclusão foram os seguintes:}

a) indivíduos com idade entre 58 e 78 anos;

b) indivíduos com diagnóstico de osteoartrite grau I de joelho, unilateral;

c) não possuir nenhum outro tipo de doença associada que acometesse os MMII (por exemplo: espondilite anquilosante, artrite reumatóide, doenças degenerativas, doenças neurológicas, Diabetes Mellitus, fraturas em tornozelo e pé, Parkinson, Paralisia Cerebral);

d) não ter realizado artroplastia de joelho ou quadril no membro estudado ou contralateral ou qualquer outro procedimento cirúrgico ortopédico nos membros inferiores;

e) não possuir nenhum distúrbio neurológico que promovesse alterações cognitivas;

f) não possuir qualquer tipo de implante metálico e/ou marcapasso;

g) não praticar atividade física regular há pelo menos 3 meses;

h) não ter realizado tratamento de fisioterapia há pelo menos 6 meses;

i) possuir tempo disponível, duas vezes na semana, para o tratamento.

\section{Os critérios de exclusão foram os seguintes:}

a) abandono do programa de tratamento antes de completar 10 sessões;

b) possuir duas faltas consecutivas;

c) apresentar hipersensibilidade aos meios físicos aplicados.

\section{MATERIAL}

Foram aplicados questionários no pré e pós tratamento fisioterapêutico para evidenciar dados pessoais e questões relacionadas a qualidade funcional do indivíduo(4) $\mathrm{com}$ a queixa principal de cada paciente.

Foram utilizados o goniômetro para medir a amplitude de movimento, a escala de dor ${ }^{(5)}$ para percepção subjetiva de dor e um esfigmomanômetro devidamente calibrado para avaliação da força muscular. ${ }^{(6)}$

A aplicação do tratamento fisioterapêutico foi realizado segundo o Padrão do IOT HCFMUSP com lençóis, bicicleta ergométrica e almofada para treino proprioceptivo, gelo triturado e o aparelho de Ondas curtas (Diatermed $\|^{\circledR}$ )

\section{MÉTODO}

Após triagem seguindo os critérios de inclusão e exclusão, cada paciente previamente informado pelo Termo de Consentimento Livre e Esclarecido aprovado pela Comissão Científica do Instituto era sorteado por um funcionário do registro para determinação do grupo de destino.

\section{Os pacientes foram divididos em três grupos:}

GRUPO A: com aplicação de ondas curtas com individuo deitado com joelhos estendidos por 20 minutos mais a realização de exercícios.

GRUPO B: com a aplicação de gelo por 20 minutos mais a realização de exercícios.

GRUPO C: somente com a realização de exercícios.

Foram formados três grupos distintos de tratamento. O primeiro grupo constou de cinesioterapia mais ondas curtas, contendo nove joelhos tratados. O segundo grupo constou de cinesioterapia mais gelo, contendo nove joelhos tratados. O terceiro grupo foi o grupo controle tratado somente com cinesioterapia, contendo sete joelhos tratados.

Os pacientes foram avaliados antes e depois do tratamento quanto à força muscular, intensidade da dor, amplitude de movimento e qualidade funcional, nos dois membros (o lado acometido e não acometido), pois os exercícios foram realizados bilateralmente e a aplicação de ondas-curtas e gelo somente no lado acometido.

A triagem e a avaliação foram realizadas por fisioterapeutas não envolvidos neste estudo (estudo cego) e todos os pacientes foram atendidos no ambulatório de Fisioterapia do IOT HCFMUSP no qual realizaram 10 sessões duas vezes por semana, com a aplicação de exercícios de alongamento e fortalecimento (isometria) para os músculos: tríceps sural, abdutores e adutores de quadril, quadríceps e isquiotibiais, propriocepção em almofada e bicicleta ergométrica e aplicação de ondas-curtas ou gelo dependendo do grupo

\section{ANÁLISE ESTATÍSTICA}

Nas comparações dos valores não paramétricos, foram utilizados os testes de Wilcoxon e Kruskal-Wallis e nas comparações dos valores paramétricos, foram utilizados os testes $t$ de student (pareado), t não pareado e a Análise de variância (ANOVA).

Nas comparações entre os grupos A, B e C para a inferência sobre a diferença entre suas médias, foi utilizada a análise de variância com discriminação das diferenças - Tukey.

Adotou-se o nível de significância de 5\% em todas as comparações e os resultados estatisticamente significantes foram indicados com asterisco $(*)$.

\section{RESULTADOS}

Os resultados foram apresentados em tabelas com os valores de média, desvio padrão e erro padrão para cada variável analisada nos três diferentes grupos no pré e pós tratamento fisioterapêutico e em quadros com os resultados estatísticos. (Tabelas 1, 2, 3, 4, Quadro 1, 2, 3, 4).

\begin{tabular}{c|c|c|c|c|c|c}
\hline Dor & \multicolumn{2}{|c|}{ Grupo A } & \multicolumn{2}{c|}{ Grupo B } & \multicolumn{2}{c}{ Grupo C } \\
\hline & Pré & Pós & Pré & Pós & Pré & Pós \\
\hline Média & 7 & 4,33 & 7,43 & 4 & 5,56 & 6,22 \\
\hline Desvio Padrão & 2,74 & 4 & 2,15 & 2,83 & 2,46 & 3,03 \\
\hline Erro Padrão & 13,04 & 30,76 & 0,81 & 1,06 & 0,81 & 1,01 \\
\hline
\end{tabular}

Tabela 1 - Valores obtidos para a variável dor.

\begin{tabular}{|l|l|l|}
\hline \multicolumn{1}{|c|}{ Comparação } & \multicolumn{1}{c|}{ Teste } & \multicolumn{1}{c|}{$\mathbf{p}$} \\
\hline \multicolumn{1}{|c|}{ Dor } & & \\
\hline A pré $\times$ A pós & Wilcoxon & 0,742 \\
\hline B pré $\times$ B pós & Wilcoxon & $0,03125^{*}$ \\
\hline C pré $\times$ C pós & Wilcoxon & 0,3672 \\
\hline A $\times$ B $\times$ C & Kruskal-Wallis & 0,3607 \\
\hline
\end{tabular}

Legenda: Grupo A: OC; Grupo B: Gelo; Grupo C: Só exercícios; * : significante

Quadro 1 - Resultados das comparações feitas entre os diferentes grupos de tratamento no pré e pós-tratamento, método estatístico utilizado e resultados obtidos para dor. 


\begin{tabular}{c|c|c|c|c|c|c}
\hline $\begin{array}{c}\text { Qualidade } \\
\text { Funcional }\end{array}$ & \multicolumn{2}{|c|}{ Grupo A } & \multicolumn{2}{c|}{ Grupo B } & \multicolumn{2}{c}{ Grupo C } \\
\hline & Pré & Pós & Pré & Pós & Pré & Pós \\
\hline Média & 11,83 & 8,89 & 15,36 & 9,50 & 15,12 & 10,89 \\
\hline Desvio Padrão & 4,16 & 6,15 & 3,42 & 3,67 & 3,65 & 3,74 \\
\hline Erro Padrão & 11,72 & 23,07 & 1,29 & 1,38 & 1,21 & 1,24 \\
\hline
\end{tabular}

Tabela 2 - Valores obtidos para a variável de qualidade funcional

\begin{tabular}{|l|l|l|}
\hline \multicolumn{1}{|c|}{ Comparação } & \multicolumn{1}{c|}{ Teste } & \multicolumn{1}{c|}{ p } \\
\hline \multicolumn{1}{|c|}{ Qualidade Funcional } & \multicolumn{1}{c|}{} \\
\hline A pré x A pós & Wilcoxon & 0,10155 \\
\hline B pré x B pós & Wilcoxon & $0,0078^{*}$ \\
\hline C pré x C pós & Wilcoxon & $0,0039^{*}$ \\
\hline A x B x C & Kruskal-Wallis & 0,9374 \\
\hline
\end{tabular}

Legenda: Grupo A: OC; Grupo B: Gelo; Grupo C: Só exercícios; * : significante

Quadro 2 - Resultados das comparações feitas entre os diferentes grupos de tratamento no pré e pós-tratamento, método estatístico utilizado e resultados obtidos para qualidade funcional.

\begin{tabular}{|c|c|c|c|c|c|c|}
\hline \multirow{2}{*}{$\begin{array}{l}\text { Flexão } \\
\text { Passiva - } \\
\text { acometido }\end{array}$} & \multicolumn{2}{|c|}{ Grupo A } & \multicolumn{2}{|c|}{ Grupo B } & \multicolumn{2}{|c|}{ Grupo C } \\
\hline & Pré & Pós & Pré & Pós & Pré & Pós \\
\hline Média & 109,78 & 114,78 & 105,14 & 113,29 & 116,44 & 126,33 \\
\hline $\begin{array}{l}\text { Desvio } \\
\text { Padrão }\end{array}$ & 13,40 & 18 & 17,38 & 14,82 & 12,28 & 13,11 \\
\hline $\begin{array}{l}\text { Erro } \\
\text { Padrão }\end{array}$ & 4,06 & 5,22 & 6,56 & 5,60 & 4,09 & 4,37 \\
\hline \multirow{2}{*}{$\begin{array}{l}\text { Flexão } \\
\text { Passiva } \\
\text { - não } \\
\text { acometido }\end{array}$} & \multicolumn{2}{|c|}{ Grupo A } & \multicolumn{2}{|c|}{ Grupo B } & \multicolumn{2}{|c|}{ Grupo C } \\
\hline & Pré & Pós & Pré & Pós & Pré & Pós \\
\hline Média & 110,33 & 115,56 & 112,29 & 118,71 & 119,33 & 129,89 \\
\hline $\begin{array}{l}\text { Desvio } \\
\text { Padrão } \\
\end{array}$ & 14,09 & 17,77 & 12,61 & 9,46 & 10,54 & 6,94 \\
\hline $\begin{array}{l}\text { Erro } \\
\text { Padrão } \\
\end{array}$ & 4,25 & 5,12 & 4,76 & 3,57 & 3,51 & 2,31 \\
\hline \multirow[t]{2}{*}{$\begin{array}{l}\text { Flexibilidade } \\
\text { - acometido }\end{array}$} & \multicolumn{2}{|c|}{ Grupo A } & \multicolumn{2}{|c|}{ Grupo B } & \multicolumn{2}{|c|}{ Grupo C } \\
\hline & Pré & Pós & Pré & Pós & Pré & Pós \\
\hline Média & 156,22 & 163,78 & 150,71 & 157,43 & 139,56 & 155,67 \\
\hline $\begin{array}{l}\text { Desvio } \\
\text { Padrão } \\
\end{array}$ & 8,09 & 12,35 & 12,50 & 8,46 & 5,81 & 12,65 \\
\hline $\begin{array}{l}\text { Erro } \\
\text { Padrão } \\
\end{array}$ & 1,71 & 2,51 & 4,72 & 3,19 & 1,93 & 4,21 \\
\hline \multirow[t]{2}{*}{$\begin{array}{l}\text { Flexibilidade } \\
\text { - não } \\
\text { acometido }\end{array}$} & \multicolumn{2}{|c|}{ Grupo A } & \multicolumn{2}{|c|}{ Grupo B } & \multicolumn{2}{|c|}{ Grupo C } \\
\hline & Pré & Pós & Pré & Pós & Pré & Pós \\
\hline Média & 158,56 & 163,11 & 161,86 & 164,57 & 143,33 & 158,89 \\
\hline $\begin{array}{l}\text { Desvio } \\
\text { Padrão } \\
\end{array}$ & 6,77 & 9,06 & 9,81 & 8,70 & 11,92 & 12,17 \\
\hline $\begin{array}{l}\text { Erro } \\
\text { Padrão }\end{array}$ & 1,42 & 1,85 & 3,70 & 3,28 & 3,97 & 4,05 \\
\hline
\end{tabular}

Tabela 3 - Valores obtidos para a variável de grau de flexão passiva e exibilidade.

\begin{tabular}{|c|c|c|}
\hline $\begin{array}{l}\text { Flexão Passiva } \\
\text { Lado acometido }\end{array}$ & Teste & $p$ \\
\hline A pré $x$ A pós & Teste $\mathrm{t}$ & 0,1108 \\
\hline B pré x B pós & Teste $\mathrm{t}$ & $0,0326^{*}$ \\
\hline C pré x C pós & Teste $\mathrm{t}$ & $0,0049^{*}$ \\
\hline$A \times B \times C$ & Anova & 0,1880 \\
\hline \multicolumn{3}{|l|}{$\begin{array}{c}\text { Flexão Passiva } \\
\text { Lado não acometido }\end{array}$} \\
\hline A pré $x$ A pós & Teste $\mathrm{t}$ & 0,1995 \\
\hline B pré x B pós & Teste $\mathrm{t}$ & $0,0150^{*}$ \\
\hline C pré x C pós & Teste $\mathrm{t}$ & $0,0407^{*}$ \\
\hline$A \times B \times C$ & Anova & 0,4721 \\
\hline \multicolumn{3}{|l|}{$\begin{array}{c}\text { Flexibilidade } \\
\text { Lado acometido }\end{array}$} \\
\hline A pré x A pós & Teste $\mathrm{t}$ & 0,0886 \\
\hline B pré $\times$ B pós & Teste $t$ & 0,1174 \\
\hline C pré x C pós & Teste $\mathrm{t}$ & $0,0019^{*}$ \\
\hline$A \times B \times C$ & Anova & 0,5115 \\
\hline \multicolumn{3}{|l|}{$\begin{array}{c}\text { Flexibilidade } \\
\text { Lado não acometido }\end{array}$} \\
\hline A pré x A pós & Teste $\mathrm{t}$ & 0,3327 \\
\hline B pré x B pós & Teste $\mathrm{t}$ & $0,0107^{*}$ \\
\hline C pré x C pós & Teste $\mathrm{t}$ & 0,1127 \\
\hline$A \times B \times C$ & Anova & 0,3424 \\
\hline
\end{tabular}

Legenda: Grupo A: OC; Grupo B: Gelo; Grupo C: Só exercícios; * * significante

Quadro 3 - Resultados das comparações feitas entre os diferentes grupos de tratamento no pré e pós-tratamento, método estatístico utilizado e resultados obtidos para amplitude de movimento de flexão e extensão passiva e flexibilidade.

\begin{tabular}{|c|c|c|c|c|c|c|}
\hline \multirow{2}{*}{$\begin{array}{c}\text { Flexores } \\
\text { - acometido } \\
\end{array}$} & \multicolumn{2}{|c|}{ Grupo A } & \multicolumn{2}{|c|}{ Grupo B } & \multicolumn{2}{|c|}{ Grupo C } \\
\hline & Pré & Pós & Pré & Pós & Pré & Pós \\
\hline Média & 62,67 & 61,44 & 73,71 & 78,57 & 50,44 & 62,22 \\
\hline Desvio Padrão & 16,67 & 10,06 & 19,91 & 22,68 & 18,49 & 17,87 \\
\hline Erro Padrão & 8,86 & 5,45 & 7,52 & 8,57 & 6,16 & 5,95 \\
\hline \multirow[t]{2}{*}{$\begin{array}{l}\text { Flexores } \\
\text { - não } \\
\text { acometido }\end{array}$} & \multicolumn{2}{|c|}{ Grupo A } & \multicolumn{2}{|c|}{ Grupo B } & \multicolumn{2}{|c|}{ Grupo C } \\
\hline & Pré & Pós & Pré & Pós & Pré & Pós \\
\hline Média & 68,56 & 61,11 & 75,71 & 80 & 54,78 & 64,44 \\
\hline Desvio Padrão & 28,10 & 11,33 & 16,18 & 8,16 & 14,75 & 12,36 \\
\hline Erro Padrão & 13,66 & 6,17 & 6,11 & 3,08 & 4,91 & 4,12 \\
\hline \multirow{2}{*}{$\begin{array}{r}\text { Extensores } \\
\text { - acometido }\end{array}$} & \multicolumn{2}{|c|}{ Grupo A } & \multicolumn{2}{|c|}{ Grupo B } & \multicolumn{2}{|c|}{ Grupo C } \\
\hline & Pré & Pós & Pré & Pós & Pré & Pós \\
\hline Média & 76,67 & 75,56 & 80 & 105,71 & 67,67 & 101,56 \\
\hline Desvio Padrão & 23,98 & 13,33 & 22,36 & 11,34 & 23,48 & 46,70 \\
\hline Erro Padrão & 10,42 & 5,88 & 8,45 & 4,28 & 7,82 & 15,56 \\
\hline \multirow{2}{*}{$\begin{array}{c}\text { Extensores } \\
\text { - não } \\
\text { acometido }\end{array}$} & \multicolumn{2}{|c|}{ Grupo A } & \multicolumn{2}{|c|}{ Grupo B } & \multicolumn{2}{|c|}{ Grupo C } \\
\hline & Pré & Pós & Pré & Pós & Pré & Pós \\
\hline Média & 77,78 & 78,89 & 90 & 95,71 & 71 & 89,11 \\
\hline Desvio Padrão & 22,79 & 19,65 & 23,09 & 22,99 & 24,29 & 27,61 \\
\hline Erro Padrão & 9,76 & 8,30 & 8,72 & 8,68 & 8,09 & 9,20 \\
\hline
\end{tabular}

Tabela 4 -Valores obtidos para a variável de grau de força muscular para os grupos flexores e extensores. 


\begin{tabular}{|c|c|c|}
\hline $\begin{array}{l}\text { Força Flexores } \\
\text { Lado acometido }\end{array}$ & Teste & $p$ \\
\hline A pré x A pós & Teste $\mathrm{t}$ & 0,3825 \\
\hline B pré x B pós & Teste $\mathrm{t}$ & 0,2448 \\
\hline C pré x C pós & Teste $t$ & 0,0584 \\
\hline$A \times B \times C$ & Anova & 0,1129 \\
\hline \multicolumn{3}{|l|}{$\begin{array}{c}\text { Força Flexores } \\
\text { Lado não acometido }\end{array}$} \\
\hline A pré $x$ A pós & Teste $\mathrm{t}$ & 0,0678 \\
\hline B pré x B pós & Teste $t$ & $0,0171^{*}$ \\
\hline C pré x C pós & Teste $\mathrm{t}$ & 0,2513 \\
\hline$A \times B \times C$ & Anova & $0,0484^{\star}$ \\
\hline$A \times B^{*}$ & Tukey & $<0,05^{*}$ \\
\hline$A \times C$ & Tukey & $>0,05$ \\
\hline$C \times B$ & Tukey & $>0,05$ \\
\hline \multicolumn{3}{|l|}{$\begin{array}{l}\text { Força Extensores } \\
\text { Lado acometido }\end{array}$} \\
\hline A pré $\times A$ pós & Teste $\mathrm{t}$ & 0,4418 \\
\hline B pré x B pós & Teste $\mathrm{t}$ & $0,0083^{*}$ \\
\hline C pré $\times C$ pós & Teste $t$ & 0,0870 \\
\hline$A \times B \times C$ & Anova & $0,1107^{*}$ \\
\hline \multicolumn{3}{|l|}{$\begin{array}{l}\text { Força Extensores } \\
\text { Lado não acometido }\end{array}$} \\
\hline A pré x A pós & Teste $t$ & 0,4059 \\
\hline B pré x B pós & Teste t & 0,4270 \\
\hline C pré $\times$ C pós & Teste $\mathrm{t}$ & 0,2515 \\
\hline$A \times B \times C$ & Anova & 0,8452 \\
\hline
\end{tabular}

Legenda: Grupo A: OC; Grupo B: Gelo; Grupo C: Só exercícios; * : significante

Quadro 4 - Resultados das comparações feitas entre os diferentes grupos de tratamento no pré e pós-tratamento, método estatístico utilizado e resultados obtidos para força muscular.

\section{DISCUSSÃO}

A prioridade desse trabalho foi determinar qual protocolo de tratamento (gelo, OC, cinesioterapia) seria mais eficaz para gerar um alívio na dor ${ }^{(5)}$, melhora na qualidade funcional(4), ganho de amplitude de movimento ${ }^{(7)}$, flexibilidade de isquiotibiais ${ }^{(8)}$ e ganho de força ${ }^{(6)}$, seguindo o sugerido pelos autores Baker e McAlindor ${ }^{(9)}$ e Fransen et al. ${ }^{(10)}$ que relataram que o mecanismo do exercício continua não sendo claro e merece estudos futuros para definir o melhor consenso de tratamento. Brandt(11) também relatou a carência de pesquisas clinicas randomizadas sobre a aplicação do calor e do frio para a melhora da condição músculo-esquelética em pacientes com OA.

Após a triagem, os pacientes enquadrados foram avaliados segundo o autor Cipriano ${ }^{(12)}$ que relata que uma história completa constitui um dos aspectos mais importantes do protocolo de avaliação clinica. A história clínica foi colhida diretamente numa avaliação pré-tratamento fisioterapêutico.

A queixa de dor do indivíduo foi avaliada através da escala CR10(5) que é uma escala de intensidade geral que pode ser utilizada para estimar a maioria dos tipos de intensidades perceptivas.

Um programa de exercícios pode ser efetivo na diminuição da dor assim como o tratamento medicamentoso com anti-inflamatórios não hormonais ${ }^{(11,13)}$. O tratamento do paciente com $\mathrm{OA}$ deve ser compreensivo com técnicas não farmacológicas consideradas de primeira escolha e analgésicos e anti-inflamatórios não hormonais como terapia adjunta ${ }^{(11)}$.

Conforme descrito por Yates ${ }^{(14)}$ e Minor ${ }^{(15)}$ os objetivos para um programa de exercícios para pacientes com OA devem ser: redução

da incapacidade e melhora da função, redução da dor articular, aumentar a ADM e flexibilidade, e facilitação da performance na realização de $A V D$ 's; proteção da articulação reduzindo os riscos de stress, atenuação das forças articulares, melhorar a biomecânica e prevenção da inabilidade e sedentarismo melhorando a forma física.

Aplicações de calor e frio, ou os dois, devem ser empregados para aliviar a dor músculo-esquelética em várias doenças, inclusive $\mathrm{OA}$. Entretanto não há controles clínicos recentes comparando a aplicação de calor e frio, em recente revisão, aproximadamente $60 \%$ dos pacientes com artrite reumatóide e OA atendidos em clínicas especializadas têm a indicação de aplicação de calor, enquanto apenas $22 \%$ relataram utilizar aplicação de gelo em articulações doloridas $^{(11)}$, sendo observado em nosso estudo, que a maioria dos pacientes avaliados utilizavam uma forma de calor (bolsa de água quente) para alívio da dor.

A diatermia por ondas curtas, que promove calor profundo, pode apresentar benefícios diminuindo a sintomatologia, porém o tratamento com diatermia é caro e precisa de estudos clínicos randomizados nos quais sua aplicação seja acompanhada por um protocolo de cinesioterapia para alongamento e fortalecimento muscular, porém Brandt(11) relata não haver evidências que tratamentos com calor são mais efetivos em analgesia do que somente exercícios.

A aplicação de calor pode produzir alívio da dor e relaxamento muscular pela elevação da temperatura subcutânea, porém é contra-indicado em pacientes com déficit circulatório, sedados, alteração de sensibilidade e casos de câncer $^{(11)}$. A aplicação de calor é uma parte limitada no programa terapêutico e deve ser usado apenas temporariamente ${ }^{(14)}$

A utilização do gelo tem a vantagem do seu baixo custo, do grande espectro de ação e da fácil aplicação técnica, mas quando um indivíduo já apresenta diminuição da sensibilidade dolorosa, é sinal de que a contração voluntária está comprometida devido ao aumento do limiar motor, decorrente do aumento da latência e da duração do potencial de ação. O gelo age diretamente no fuso muscular e no órgão tendinoso, portanto uma sobrecarga na execução de exercícios após resfriamento do músculo, pode levar a uma nova lesão muscular, uma vez que o controle motor está com o seu limiar alterado ${ }^{(16)}$, devido a isso nos baseamos em um programa de exercícios que não causasse danos musculares aos indivíduos.

A variável dor obteve uma melhora significativa somente no grupo B. Este trabalho demonstrou que o tratamento utilizando somente a cinesioterapia não foi benéfico na variável dor, porém apresentou resultados positivos na melhora da qualidade funcional, ganho de amplitude de movimento e ganho de força muscular, contrariando Brandt ${ }^{\left({ }^{11)}\right.}$ e Fransen et al. ${ }^{(10)}$ que relataram que somente exercícios terapêuticos em indivíduos com OA de quadril e joelho reduzem a dor e melhoram a atividade funcional.

Estudo realizado por Teixeira e Olney ${ }^{(17)}$ mostrou a existência de correlação entre dor e rigidez articular e entre dor e função. Este achado sugere que o alívio da dor está diretamente relacionado com ganhos da função, sendo, portanto um objetivo importante a ser considerado no tratamento de pacientes com OA. Estes sugerem que uma abordagem terapêutica, visando o alívio da dor e redução da rigidez articular, é fundamental para que os pacientes com OA apresentem um melhor empenho funcional.

Jitpraphai et al. ${ }^{(18)}$ realizaram um estudo em pacientes com OA de joelhos e aplicam um tratamento utilizando calor e exercícios encontrando que exercícios são apropriados por serem fáceis, de baixo custo e seguros. Obtiveram melhora nos sintomas de dor e na capacidade para caminhar, sendo apenas $7.66 \%$ dos pacientes submetidos a procedimentos cirúrgicos.

O Índice Algo Funcional de Lequesne ${ }^{(4,19)}$ juntamente com o Índice Algo Funcional de WOMAC apresentam a eficácia para a avaliação da qualidade de vida em pacientes portadores de OA, porém em nosso estudo preferimos a utilização do Índice de Lequesne ${ }^{(4,19)}$ por ser validado para a língua portuguesa. A qualidade de vida 
mensurada com este índice obteve resultados positivos em todos os grupos, porém só estatisticamente significante no grupo B e no grupo C, no entanto, esta melhora não apresentou diferenças quando comparadas entre os três grupos, mostrando os benefícios da realização de exercícios.

A utilização da crioterapia para o aumento da amplitude de movimento articular ainda é incerta. O aumento do limiar da dor e a diminuição da velocidade de condução nervosa beneficiam o alongamento muscular, em contrapartida, a diminuição da extensibilidade do tecido conectivo atua reduzindo a flexibilidade muscular ${ }^{(16)}$.

A amplitude de movimento teve resultados positivos quando comparados pré e pós-tratamento com significância no grupo B e no grupo $\mathrm{C}$ tanto para o lado acometido como para o não acometido, quando comparados os três grupos pela análise de variância esta melhora não apresentou diferença estatisticamente significativa.

Houve ganho de flexibilidade em todos os grupos, porém sendo significativo quando comparados pré e pós-tratamento no grupo C somente no joelho acometido e no grupo B somente no joelho não acometido, quando comparados os três grupos pela análise de variância esta melhora não apresentou diferenças. Estes resultados são discutíveis uma vez que em todos os joelhos não acometidos foram realizados cinesioterapia e não existem estudos que avaliem essa variável para eventual comparação.

A avaliação da força muscular é uma técnica importante para diagnosticar a etiologia da doença e para definir e avaliar estratégias de reabilitação. Tradicionalmente a força muscular tem sido avaliada através do teste muscular manual e esta técnica tem sido criticada devido à sua natureza subjetiva. Com o desenvolvimento de dinamômetros tornou-se possível medidas objetivas de torque muscular, sendo este o melhor método para avaliação de força muscular(17).

Por não ser possível a utilização do dinamômetro isocinético devido ao seu alto custo a força muscular da musculatura de joelho foi avaliada segundo o descrito por Bastone, modificado e validado por Helewa et al. ${ }^{(20)}$ em 1981.

Reilly et al.(21), após avaliar 300 indivíduos entre homens e mulheres com idade entre 40 e 79 anos, concluíram que a fraqueza do músculo quadríceps está diretamente associada a dor articular no joelho e desabilidade da articulação, o que foi verificado em nosso estudo durante as avaliações pré-tratamento.

A OA resulta em alterações que afetam não só tecidos intracapsulares, como também tecidos periarticulares, como os ligamentos, cápsula, tendões e músculos. Pacientes com OA quando comparados com indivíduos saudáveis de mesma idade apresentaram fraqueza do músculo quadríceps, redução da propriocepção de joelho e diminuição do equilíbrio e senso de posição( ${ }^{(13,22)}$.

Wilson e Mayer(23) referiram que a presença de efusão articular, mesmo em pequena quantidade, é um potente mecanismo inibitório da atividade muscular reflexa daquela articulação. A diminuição da atividade muscular reflexa causa hipotrofia e fraqueza precocemente, com os conseqüentes prejuízos mecânicos associados ${ }^{(24)}$.

Booth ${ }^{(25)}$ referiu que a força muscular declina rapidamente durante a imobilização de um membro por diminuição do tamanho do músculo e da tensão por unidade de área transversa do músculo. A grande perda absoluta de massa muscular ocorre no inicio do processo de hipotrofia(24).

Segundo Greve et al. ${ }^{(24)}$ a dor inibe a atividade muscular reflexa, causando hipotrofia e fraqueza muscular, o processo doloroso é prévio ao quadro de fraqueza muscular, essa afirmação sugere que exercícios são benéficos para ganho de força e conseqüentemente melhora na dor.

Os benefícios dos exercícios aeróbicos incluem o aumento da capacidade aeróbica, alongamento e fortalecimento muscular, resistência e perda de peso. Os mais indicados são a caminhada, ciclismo, natação, dança, e hidroginástica. Os exercícios isométricos são geralmente preferidos no início do tratamento do que os isotônicos ${ }^{(11,13)}$.

Em estudo randomizado controlado realizado por Brandt ${ }^{(11)}$ e Bischoff e Ross ${ }^{(13)} \mathrm{com}$ pacientes com OA moderada, que realizaram fortalecimento muscular com isometria obtiveram uma significante diminuição da dor articular, e pacientes com avançada OA, após oito semanas de realização de isometria de quadríceps permitem a redução da dose diária de analgésicos.

Este estudo demonstrou a manutenção da intensidade da força aferida da musculatura flexora de joelho nos grupos A e B nos joelhos acometidos e no grupo B nos joelhos não acometidos, em contrapartida houve ganho de força nos indivíduos do grupo C nos joelhos acometidos e não acometidos e perda de força nos joelhos não acometidos no grupo $A$. Em relação a musculatura extensora do joelho, foi observado ganho de força no grupo B e C nos joelhos acometidos e perda de força no grupo A. Nos joelhos não acometidos, houve ganho de força no grupo $\mathrm{C}$, perda de força no grupo $B$ e manutenção do grupo $A$.

Infere-se assim, que o ganho de força para a musculatura flexora e extensora do joelho não deve estar relacionado a aplicação de gelo ou OC. A divergência dos dados pode ser em decorrência do método de avaliação utilizado, duração de tratamento preconizado neste trabalho ou do protocolo de tratamento para ganho de força que se manteve durante todo o período não havendo aumento de carga na realização dos exercícios, contrariando os autores Kisner e Colby(26) que afirmam ser necessário o aumento de carga progressiva para significativo ganho de força.

Acreditamos que novos estudos clínicos prospectivos sejam realizados para validação do método de aferição de força utilizando esfigmomanômetro e aplicação deste protocolo adaptando o tempo de tratamento e a carga utilizada.

\section{CONCLUSÕES}

1. O protocolo mais adequado de tratamento para alivio da dor foi o que envolveu a aplicação de gelo e cinesioterapia.

2. O nível de qualidade funcional melhorou nos três grupos estudados.

3. O ganho de amplitude de movimento, flexibilidade e força muscular não possui relação de melhora que envolva a apliçação de gelo e calor. 


\section{REFERÊNCIAS BIBLIOGRÁFICAS}

1. Greve JMA, Plapler PG, Seguchi HH, Pastore EH, Battistella LR. Tratamento fisiátrico da dor na osteoartrose. Rev Hosp Clin Fac Med Univ São Paulo. 1992; 47:185-9.

2. Mühlen CA. Osteoartrose: como diagnosticar e tratar. Rev Bras Med. 2000; 57(3):150-5.

3. Camanho GL. Tratamento da osteartrose do joelho. Rev Bras Ortop. 2001; 36:135-40.

4. Borg G. Escalas de Borg para a dor e o esforço percebido. São Paulo: Manole; 2000

5. Bastone AC. Impacto da atividade física no desempenho funcional do idoso institucionalizado [dissertação]. São Paulo: Faculdade de Medicina, Universidade de São Paulo; 2000.

6. Lequesne M, Mery C, Sanson M, Gerard P. Indexes of severity for osteoarthritr of the hip and knee.Validation-value in comparison eith other in comparison. Scand J Rheumatol. 1987; 65:85-9.

7. Marques AP. Manual de goniometria. São Paulo: Manole; 1997

8. Mangine RE. Physical therapy of the knee. 2th ed. New York: Churchill Lingstone; 1995.

9. Baker KB, Mcalindon T. Exercise for knee osteoarthritis. Curr Opin Rheumatol. 2000; 12: 456-63.

10. Fransen M, McConnell S, Bell M. Therapeutic exercise for people with osteoarthritis of the hip or knee. A systematic review. J Rheumatol. 2002; 29:1737-45.

11. Brandt KD. The importance of nonpharmacologic approaches in management of osteoarthritis. Am J Med. 1998; 105(1B): 39S -44S.

12. Cipriano JS. Manual fotográfico de testes ortopédicos e neurológicos. 3 ed. São Paulo: Manole; 1999.

13. Bischoff HA, Roos EM. Effectiveness and safety of strengthening, aerobic, and coordination exercises for patients with osteoarthritis. Curr Opin Rheumatol. 2003; 15:141-4

14. Yates DAH. The treatment of osteoarthorsis. Practitioner. 1972; 208:43-7.
15. Minor M. Exercise in the management of osteoarthritis of the knee and hip. Arthritis Care Res. 1994; 7:198-204.

16. Guirro R, Abib C, Máximo C. Os efeitos fisiológicos da crioterapia: uma revisão. Rev Fisioter Univ São Paulo. 1999; 6:164-70.

17. Teixeira LF, Olney SJ. Avaliação clinica, radiológica e estudo isocinético da força muscular em pacientes idosos portadores de osteoartrite de joelho. Rev Fisioter Univ São Paulo. 1995; 2:56-64.

18. Jitpraphai $\mathrm{C}$, Cheamvaraporn $\mathrm{K}$. Conservative management of degenerative knee: an experience with 508 cases at ramathibodi hospital. J Med Assoc Thai. 1992; 75:35-8

19. Lequesne M. Indices of severity and disease activity for ostearthritis. Semin Arthritis Rheum. 1991; 20(6Suppl 2):48-54

20. Helewa A, Goldsmith $\mathrm{CH}$, Smythe HA. The modified sphygmomanometer-an instrument to measure muscle strength: validation study. J Chronic Dis. 1981; 34:353-61.

21. O'Reilly SC, Jones A, Muir KR, Doherty M. Quadriceps weakness in knee ostearthritis: the effect on pain and disability. Ann Rheum Dis. 1998; 57:588-94

22. Hassan BS, Mockett S, Doherty M. Static postural sway, proprioception, and maximal voluntary quadriceps contraction in patients with knee osteoarthritis and normal control subjects. Ann Rheum Dis. 2001; 60:612-8.

23. Wilson $\mathrm{CH}$ Jr, Mayer WP. Exercise and mobilization techniques in principles of physical medicine and rehabilitation in the muscles diseases. Orlando: Grune \& Stratton; 1986

24. Greve JMD, Plapler PG, Seguchi HH, Pastore EH, Battistella LR. Cinesioterapia na osteoartrose. Med Reabil. 1992; 31:5-9.

25. Booth FW. Physiologic and biochemical effects of immobilization on muscle. Clin Orthop Relat Res.1987; (219):15-20

26. Kisner C, Colby LA. Exercícios terapêuticos: fundamentos e técnicas. 3a. ed São Paulo: Manole; 1998. 